21st Particles and Nuclei International Conference (PANIC 2017)

International Journal of Modern Physics: Conference Series

Vol. 46 (2018) 1860001 (9 pages)

(C) The Author(s)

DOI: $10.1142 / S 2010194518600017$

\title{
Neutrino Physics with Nuclear Reactors: An Overview
}

\author{
J. P. Ochoa-Ricoux \\ Instituto de Física, Pontificia Universidad Católica de Chile \\ Avda. Vicuña Mackenna 4860, Santiago, Chile \\ jpochoa@uc.cl
}

Published 3 May 2018

\begin{abstract}
Nuclear reactors provide an excellent environment for studying neutrinos and continue to play a critical role in unveiling the secrets of these elusive particles. A rich experimental program with reactor antineutrinos is currently ongoing, and leads the way in precision measurements of several oscillation parameters and in searching for new physics, such as the existence of light sterile neutrinos. Ongoing experiments have also been able to measure the flux and spectral shape of reactor antineutrinos with unprecedented statistics and as a function of core fuel evolution, uncovering anomalies that will lead to new physics and/or to an improved understanding of antineutrino emission from nuclear reactors. The future looks bright, with an aggressive program of next generation reactor neutrino experiments that will go after some of the biggest open questions in the field. This includes the JUNO experiment, the largest liquid scintillator detector ever constructed which will push the limits of this detection technology.
\end{abstract}

Keywords: Reactor; neutrino; overview.

\section{Introduction}

Neutrinos are fascinating elementary particles with unique properties that may help unravel some of the secrets of the universe. Not only are they invaluable messengers that can travel for light-years without interference, but they are also extremely abundant. Additionally, they behave in contradiction with the Standard Model (SM), and can thus guide the way to new theories.

The underlying mechanism for neutrino oscillation is the mixing between the weak eigenstates $\left(\nu_{e}, \nu_{\mu}, \nu_{\tau}\right)$ and the mass eigenstates $\left(\nu_{1}, \nu_{2}, \nu_{3}\right)$. This mixing is parameterized by a $3 \times 3$ unitary matrix known as Pontecorvo-Maki-NakagawaSakata (PMNS), ${ }^{1,2}$ which is typically parameterized in terms of four parameters:

This is an Open Access article published by World Scientific Publishing Company. It is distributed under the terms of the Creative Commons Attribution 4.0 (CC-BY) License. Further distribution of this work is permitted, provided the original work is properly cited. 


\section{J. P. Ochoa-Ricoux}

three mixing angles $\left(\theta_{12}, \theta_{23}, \theta_{13}\right)$, and one CP-violating phase $\delta .^{\text {a }}$ From this formalism it is possible to calculate the probability that a neutrino created under a given flavor will "disappear" (oscillate) into others. For example, the survival probability in the plane-wave approximation for an electron antineutrino with energy $E$ after traveling a distance $L$ is given by

$$
\begin{aligned}
P_{\bar{\nu}_{e} \rightarrow \bar{\nu}_{e}} & =1-\cos ^{4} \theta_{13} \sin ^{2} 2 \theta_{12} \sin ^{2} \Delta_{21} \\
& -\sin ^{2} 2 \theta_{13}\left(\cos ^{2} \theta_{12} \sin ^{2} \Delta_{31}+\sin ^{2} \theta_{12} \sin ^{2} \Delta_{32}\right),
\end{aligned}
$$

where $\Delta_{j i} \equiv 1.267 \Delta m_{j i}^{2}\left(\mathrm{eV}^{2}\right) \frac{L(\mathrm{~m})}{E(\mathrm{MeV})}$, and $\Delta m_{j i}^{2}=m_{j}^{2}-m_{i}^{2}$ is the difference between the mass-squares of the $j$-th and $i$-th mass eigentates.

A vast body of experimental evidence for neutrino oscillation has accumulated in the last two decades, ${ }^{3}$ and all the mixing angles and mass splittings are now known with a $<10 \%$ precision. ${ }^{4}$ Past reactor neutrino experiments have played a key role in the quest to understand the neutrino, from its discovery ${ }^{5}$ to the clear $L / E$ disappearance signature first observed at KamLAND. ${ }^{6}$ Despite the great progress, many open questions remain, such as the origin of neutrino masses and their absolute values. It is also not known if the third neutrino mass eigenstate is heavier or lighter than the other two, i.e. if the mass hierarchy is normal or inverted respectively. Finally, it is not known if neutrinos obey the CP symmetry or if there are additional generations beyond the known three, among many other questions.

Reactor neutrino experiments are at the forefront for answering some of these questions. The remainder of this article is divided into three main sections. The first one describes the basic principles and characteristics of reactor neutrino experiments. The second one focuses on the latest results obtained by ongoing experiments, as well as the anomalies that they have uncovered. The third and last section focuses on the prospects for the future. It should be noted that, due to space constraints, this work does not represent an exhaustive review, but rather a selection driven by the personal interests of this author.

\section{Essentials of Reactor Neutrino Experiments}

Nuclear power plants are an extremely high-yielding, well-understood, and pure source of electron antineutrinos. These particles are emitted as the neutron-rich fission products in the reactor core undergo beta decay $\left(n \rightarrow p+e^{-}+\bar{\nu}_{e}\right)$ towards stability. In average, about 6 antineutrinos are emitted per fission, for a total rate of about $2 \times 10^{20} \bar{\nu}_{e} / \mathrm{s}$ for a $1 \mathrm{GW}_{\text {th }}$ reactor. Electron antineutrinos are produced primarily through the fissions of 4 main isotopes: ${ }^{235} \mathrm{U},{ }^{239} \mathrm{Pu},{ }^{238} \mathrm{U}$, and ${ }^{241} \mathrm{Pu}$. Knowing the fractions that each of these four isotopes contribute to the total fissions at a given time, as well as the total reactor power, it is possible to predict the expected antineutrino flux.

${ }^{a}$ If neutrinos are Majorana particles, two extra complex phases are part of the parameterization. 
As shown by Eq. 1, the disappearance of electron antineutrinos gives access to most neutrino oscillation parameters $\left(\theta_{13}, \theta_{12}, \Delta m_{21}^{2}\right.$ and $\left.\Delta m_{32}^{2}\right)$ in a way that is independent of $\theta_{23}$ and $\mathrm{CP}$ effects. The primary detection channel in these experiments is the inverse beta-decay (IBD) reaction $\left(\bar{\nu}_{e}+p \rightarrow e^{+}+n\right)$. In addition to its large cross-section compared to other neutrino interactions, this reaction allows for powerful background rejection thanks to the coincidence between the positron and neutron signals. Moreover, the energy of the positron preserves most of the information about the incoming antineutrino energy.

\section{Ongoing Experiments}

\subsection{Experimental Setup}

The three largest reactor neutrino experiments currently in operation, Double CHOOZ, RENO and Daya Bay, were built with the same primary purpose in mind: making a precise measurement of the $\theta_{13}$ mixing angle. This is done by sampling the $\bar{\nu}_{e}$ flux at a near location(s), where oscillation effects are small, and at a far location(s), where the oscillation effects modulated by $\theta_{13}$ are maximal. Identically designed detectors are used in order to obtain a first order cancellation of the uncertainties in the flux prediction and the detection efficiencies.

Some of the main parameters of these experiments are compared in Table 1. While Double CHOOZ and RENO each use one near detector and one far detector, Daya Bay uses an array of eight detectors spread amongst two near sites and one far site, as shown on the left of Fig. 1. The three experiments use very similar detection technologies, consisting of three nested volumes separated by acrylic and surrounded by an array of photomultiplier tubes (PMTs). From the innermost to the outermost, these volumes contain gadolinium-doped liquid-scintillator (Gd-LS), pure LS, and Mineral Oil (MO). The Gd-LS constitutes the antineutrino target, with the gadolinium serving as the primary capture agent for IBD neutrons with a clear gamma signature at $\sim 8 \mathrm{MeV}$. The LS allows to better measure the energy of those events occurring near the target's boundary, and the MO shields against the radioactivity coming mainly from the vessel and the PMTs' glass. The detectors are immersed in instrumented water pools, which provide additional shielding and serve as cosmic-ray muon detectors. A diagram illustrating these principles in the case of Daya Bay is shown on the right of Fig. 1. More details about the three experiments can be found in Refs. 7, 8, 9 .

Table 1. Comparison of some of the parameters of the Double CHOOZ, RENO and Daya Bay experiments. $P_{\text {th }}$ stands for the total reactor thermal power.

\begin{tabular}{c|c|c|c|c}
\hline & $\mathrm{P}_{\mathrm{th}}[\mathrm{GW}]$ & $\begin{array}{c}\text { nGd target mass } \\
\text { @ far site [tons] }\end{array}$ & $\begin{array}{c}\text { Overburden } \\
\text { (near/far) [mwe] }\end{array}$ & $\begin{array}{c}\text { Data-taking } \\
\text { (start-end) }\end{array}$ \\
\hline Double Chooz & 8.6 & 8.3 & $80 / 300$ & $2011-2017$ \\
RENO & 16.4 & 15.4 & $90 / 440$ & $2011-2021$ \\
Daya Bay & 17.4 & 80 & $270 / 950$ & $2011-2020$
\end{tabular}




\section{J. P. Ochoa-Ricoux}
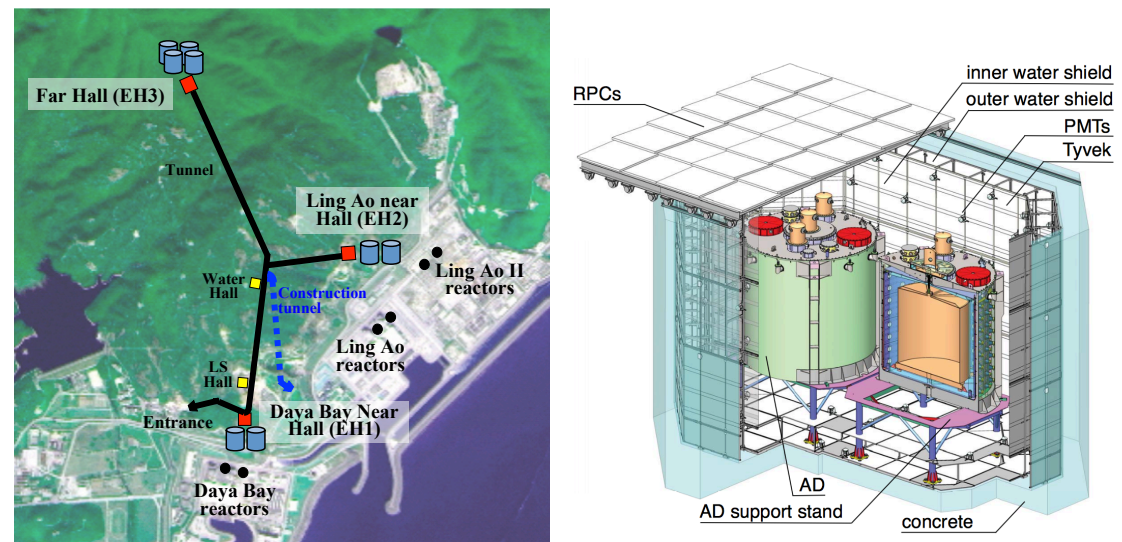

Fig. 1. (Left) Schematic layout of the Daya Bay Experiment. A total of eight identically designed antineutrino detectors, represented as cylinders, are deployed in the three experimental halls (EHs). The six reactor cores are represented by the black dots. (Right) Cross-section of all detector systems in a Daya Bay near site. A cross-section of one of the two ADs is shown to illustrate the three nested volumes described in the text. Figure obtained from Ref. 10.

\subsection{Oscillation Physics Results}

After some hints for a non-zero $\theta_{13},{ }^{11,12,7}$ the first definite observation at the level of $5 \sigma$ came from the Daya Bay experiment in $2012,{ }^{13}$ followed by a confirmation from RENO. ${ }^{8}$ The measurement has been refined since, and rate and spectral shape analyses done by Daya Bay ${ }^{10}$ and $\mathrm{RENO}^{14}$ have also yielded precise estimates of $\Delta m_{32}^{2}$. The global landscape for these two parameters at the time of this conference is shown in Fig. 2. The value of $\sin ^{2} 2 \theta_{13}$ is now known to better than $4 \%$. Similarly, the precision of $\sim 3 \%$ obtained for $\Delta m_{32}^{2}$ by Daya Bay matches that of accelerator experiments.

The precise $\bar{\nu}_{e}$ disappearance signature measured in these experiments also allows to search for sterile neutrino mixing. In the minimal extension to the
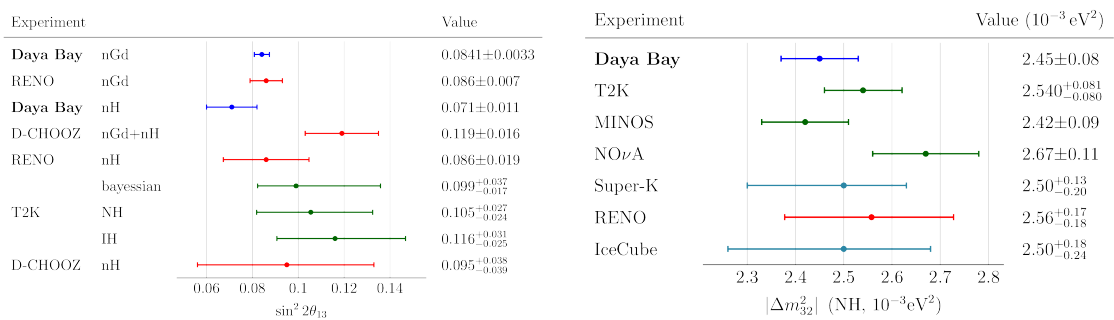

Fig. 2. (Left) Comparison of $\theta_{13}$ measurements. (Right) Comparison of $\Delta m_{32}^{2}$ measurements, under the assumption of the normal hierarchy $(\mathrm{NH})$. Both figures represent the state of the art at the time of this conference. Figure courtesy of M. Gonchar. 
three-flavor neutrino model, where only a fourth sterile neutrino is added to the three active ones, the search essentially amounts to looking for an additional spectrum distortion with a frequency $\Delta m_{41}^{2}$ distinct to $\Delta m_{32}^{2}$. With its configuration of multiple baselines from three experimental sites to three groups of nuclear reactors, Daya Bay is particularly well suited for this measurement. The latest results from Daya Bay were recently combined with those from the MINOS experiment in order to probe the hints for electron antineutrino appearance observed by the LSND and MiniBooNE experiments. ${ }^{15}$ As shown on the left of Fig. 3, the resulting limits span over six orders of magnitude in $\Delta m_{41}^{2}$ and exclude the parameter space allowed by LSND and MiniBooNE for $\Delta m_{41}^{2}<0.8 \mathrm{eV}^{2}$ at $95 \%$ C.L. RENO has also released a preliminary result using only their data. ${ }^{16}$

\subsection{Comparison with Reactor Models}

In 2011, the comparison between the measured flux from nuclear reactors and the state-of-the-art prediction based on the Huber-Mueller model ${ }^{18,19}$ resulted in the socalled "reactor antineutrino anomaly" (RAA). ${ }^{20}$ This anomaly consists in a $\sim 2.5 \sigma$ deficit of the average flux measured by previous short baseline reactor experiments with respect to the expectation, as shown on the right of Fig. 3. The most exciting explanation is the existence of an $\mathrm{eV}$-scale sterile neutrino, although unaccounted systematics and/or biases in the prediction could also be partially or totally at fault. As seen in Fig. 3, the latest results from Daya Bay have confirmed the RAA. Preliminary results from $\mathrm{RENO}^{21}$ are also in agreement.

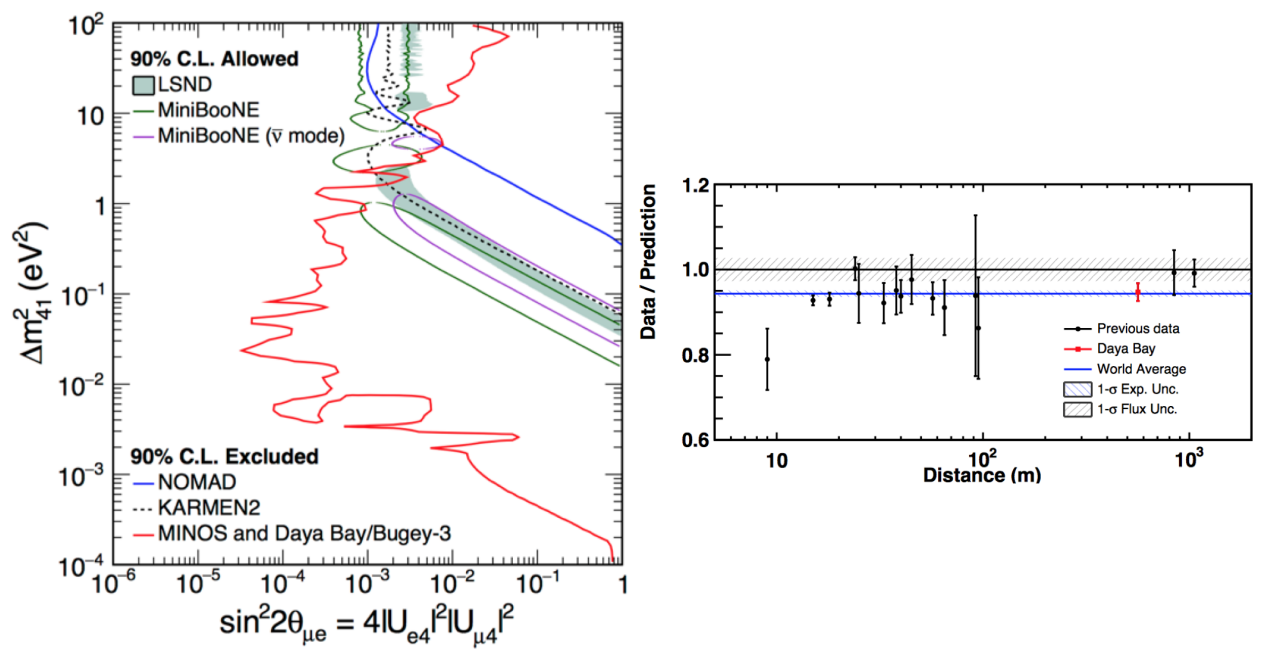

Fig. 3. (Left) MINOS and Daya Bay + Bugey-3 combined $90 \%$ exclusion limit, with the LSND and MiniBooNE allowed regions, from Ref. 15. (Right) Measured $\bar{\nu}_{e}$ rate vs. Huber-Mueller model prediction for Daya Bay (red) and previous short baseline reactor experiments (black), from Ref. 17. 


\section{J. P. Ochoa-Ricoux}

The unprecedentedly high-statistics of ongoing reactor experiments have allowed to extend the comparison between observation and prediction to other facets. The left of Fig. 4 shows the expected spectral shape of reactor antineutrinos from the Huber-Mueller model and the observation made at Daya Bay, showing a localized discrepancy between $4-6 \mathrm{MeV}$ with a $>4 \sigma$ significance. This additional spectral "anomaly" has also been seen by Double CHOOZ, ${ }^{22} \mathrm{RENO}^{14}$ and NEOS, ${ }^{23}$ and cannot be fully explained by sterile neutrino oscillations. The excess has been found to be correlated with reactor power, disfavoring any unknown background. Moreover, it has not been seen in other spectra (e.g. $\left.{ }^{12} \mathrm{~B}\right)$, ruling out an effect caused by electronics or the energy model.

Likewise, a novel measurement from Daya Bay that studies how the flux and spectral shape of reactor antineutrinos changes as a fuel cycle progresses sheds additional light on these discrepancies. ${ }^{24}$ The analysis is done in terms of the effective fission fraction $F_{239}$, defined as the percentage of total fissions from the ${ }^{239} \mathrm{Pu}$ isotope. This quantity increases in a fuel cycle as the corresponding quantity for ${ }^{235} \mathrm{U}$, $F_{235}$, decreases. The right of Fig. 4 shows how the evolution of the total IBD yield as a function of $F_{239}$ is different from the Huber-Mueller model's expectation at the $3.1 \sigma$ level. From these data it is possible to extract information on the individual IBD yields for the major fissioning isotopes $\left({ }^{235} \mathrm{U}\right.$ and $\left.{ }^{239} \mathrm{Pu}\right)$ while making conservative assumptions on the minor ones $\left({ }^{241} \mathrm{Pu}\right.$ and $\left.{ }^{238} \mathrm{U}\right)$. The results show that while the individual yield for ${ }^{239} \mathrm{Pu}$ is in good agreement with the expectation, there is a significant tension in the ${ }^{235} \mathrm{U}$ case. The hypothesis that all isotopes contribute

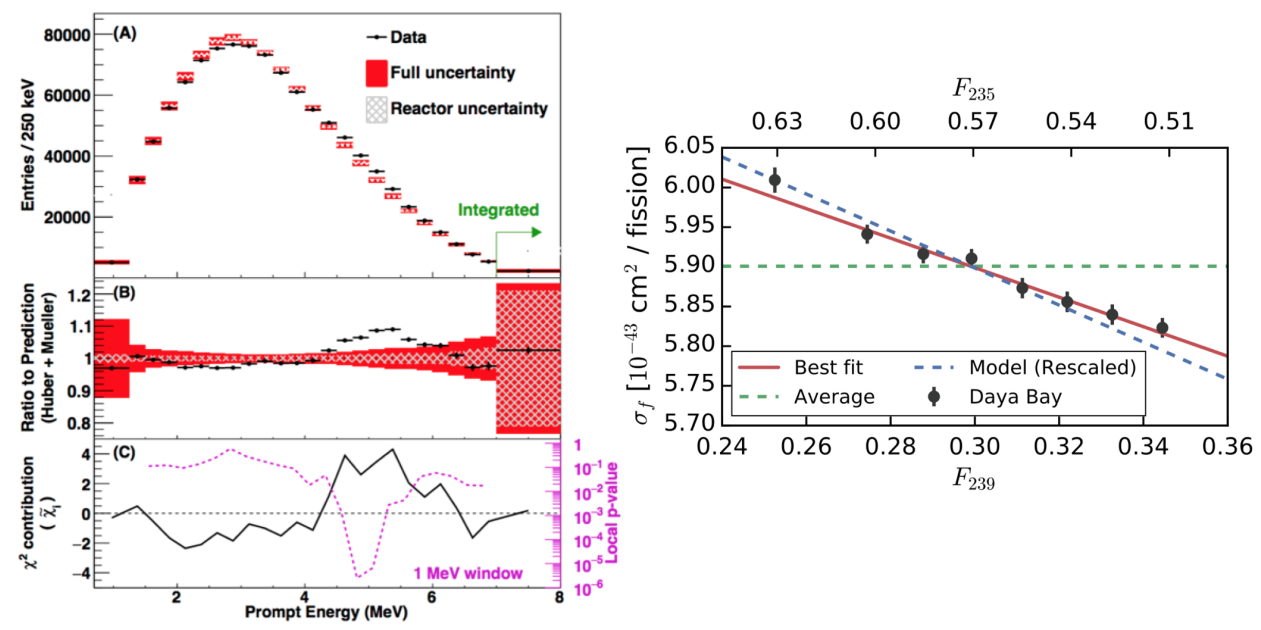

Fig. 4. (Left) Comparison of predicted and measured prompt energy spectra of nGd events, after normalization to the number of events. The ratio, $\chi^{2}$ distribution of each bin, and local p-values for $1 \mathrm{MeV}$ energy windows, are also shown. (Right) Measured and predicted total IBD yield per fission $\sigma_{f}$ vs. $F_{239}$. 
equally to the RAA is disfavored at the $2.8 \sigma$ level, weakening the pure sterile neutrino interpretation. A similar study is in progress by the RENO Collaboration. ${ }^{21}$ Together, the flux, spectral shape, and fuel evolution discrepancies strongly suggest that unaccounted systematics, or other problems with the prediction, are at least partially at play.

\section{Future Prospects}

A bright outlook is on the horizon for reactor neutrino experiments. On the one hand, running experiments still have much to contribute. Double CHOOZ just terminated reactor data collection in December 2017, and a recount of the number of target protons during disassembly will allow to reduce their most important systematic uncertainty. RENO is looking for a possible extension to 2021, and aims for a $\sim 6 \%$ precision in both $\theta_{13}$ and $\Delta m_{32}^{2}$. Daya Bay will run until 2020, at which point a precision of $<3 \%$ in both of these parameters is foreseen. Many other new and improved results are also expected from these experiments in the next few years.

In addition, an aggressive experimental program of short baseline reactor experiments conceived to shed light on the anomalies presented in Sec. 3.3 is underway. A comprehensive review of this program can be found in Ref. 25. Many of the experiments have movable and/or segmented detectors, allowing to search for oscillations to an eV-scale sterile neutrino at different baselines. Also, by virtue of their location near a highly-enriched uranium (HEU) reactor, some of these experiments can independently constrain the ${ }^{235} \mathrm{U}$ yield, allowing to further scrutinize the prediction models and to directly explore the question of whether the 4-6 MeV shape anomaly is the same for all isotopes. Results from some of these projects are starting to pour in, and a surprise could be in store during the next year or two.

Finally, a major reactor antineutrino experiment, the Jiangmen Underground Neutrino Observatory (JUNO), is being constructed. Its primary purpose is the determination of the neutrino mass hierarchy through the measurement of the subdominant $\Delta m_{32}^{2}$-driven fast oscillation that runs alongside the $\Delta m_{21}^{2}$-driven slow oscillation. As shown on the left of Fig. 5, the choice of neutrino mass hierarchy introduces phase shifts to these subdominant oscillations, whose discriminatory power is maximized when the $\Delta m_{21}^{2}$-driven oscillations are also maximal. Accordingly, JUNO will be located at the strategic distance of $53 \mathrm{~km}$ from ten reactors in the southeast of China. Sensitivity studies show that JUNO will determine the mass hierarchy at the level of $3-4 \sigma$ in a period of about 6 years. The experiment will also be able to measure $\sin ^{2}\left(2 \theta_{12}\right), \Delta m_{21}^{2}$ and $\Delta m_{32}^{2}$ to better than $1 \%$, and has a rich program with supernova neutrinos, geo-neutrinos, solar neutrinos and atmospheric neutrinos. Finally, it will be a good detector to search for proton decay and other new physics phenomena. More details on JUNO's physics program can be found in Ref. 26.

In order to accomplish these aggressive goals, JUNO will have to be the largest and most precise liquid scintillator detector ever attempted in history. A schematic 

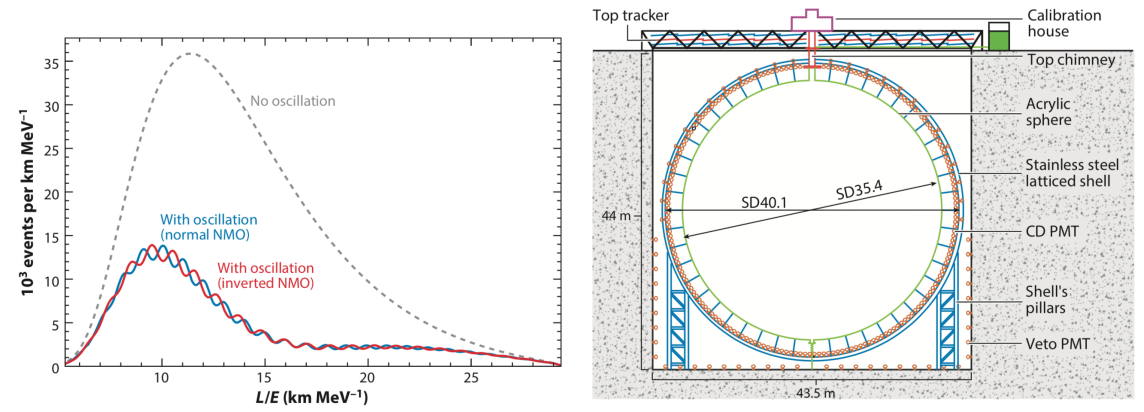

Fig. 5. (Left) Predicted electron antineutrino spectrum at JUNO with infinite statistics and perfect energy resolution, as a function of neutrino travel distance L over energy E. (Right) Schematic of the JUNO detector. Both plots were obtained from Ref. 27.

illustrating the detector's conceptual design is shown on the right of Fig. 5. The central detector (CD) will consist of a $\sim 35 \mathrm{~m}$ acrylic sphere containing $20 \mathrm{ktons}$ of liquid scintillator. An exquisite energy resolution of $3 \%$ at $1 \mathrm{MeV}$ is required for the measurement of the mass hierarchy, accomplished primarily through the use of a high transparency \& high light-yield LS, and an array of 18, 000 large (20 inch) PMTs providing $75 \%$ optical coverage. A mixture of microchannel plate and dynode PMTs will be used, both with an unprecedentedly high detection efficiency of $\sim 30 \%$. The space between the large PMTs will be filled with 25,000 small (3 inch) PMTs, whose main function will be to help calibrate the large PMT system by virtue of their operation in photon-counting mode. The acrylic sphere and the surrounding stainless steel shell will be immersed in an instrumented ultrapure water pool, serving as a shield and as a muon veto detector.

Civil construction for JUNO began in 2015 and is well advanced. The first PMTs have already been produced and tested. So far everything is on track for an on time start of data-taking in late 2020 .

\section{Summary \& Conclusions}

Reactor neutrino experiments have traditionally played a key role in unveiling the secrets of the neutrino, and everything seems to indicate that this trend will continue. Their successful determination of the $\theta_{13}$ mixing angle a few years ago was a key discovery that opened the door to measuring the mass hierarchy with neutrino oscillations and to searching for $\mathrm{CP}$ violation in the leptonic sector. In addition to being able to determine the parameters that drive electron antineutrino disappearance at short baselines with the best precision in the world, current reactor experiments also lead the way in the search for light sterile neutrinos. Ongoing experiments have also revealed a number of discrepancies with reactor models, whose resolution will culminate in new physics and/or in a deeper understanding of the physics of antineutrino emission from nuclear reactors. The future looks bright, with a vast 
array of experiments searching for sterile neutrinos at short baselines that are just now starting to come online. Likewise, the JUNO experiment will be the largest and most precise liquid scintillator detector ever constructed, with a rich program in neutrino physics and astrophysics elapsing over two decades.

\section{Acknowledgements}

The author would like to thank the National Commission for Scientific and Technological Research of Chile (CONICYT) for its support. This work was supported by funds from the FONDECYT ${ }^{\mathrm{b}}$ program, grant number 11130530.

\section{References}

1. B. Pontecorvo, Sov. Phys. JETP 6, p. 429 (1957).

2. Z. Maki and M. Nakagawa and S. Sakata, Progress of Theoretical Physics 28, 870 (1962).

3. C. Giganti, S. Lavignac and M. Zito, Prog. Part. Nucl. Phys. 98, 1 (2018).

4. C. Patrignani et al., Chin. Phys. C40, p. 100001 (2016).

5. C. Cowan, F. Reines, F. Harrison, H. Kruse and A. McGuire, Science 124, 103 (1956).

6. K. Eguchi et al. (KamLAND Collab.), Phys.Rev.Lett. 90, p. 021802 (2003).

7. Y. Abe et al. (Double CHOOZ Collab.), Phys.Rev.Lett. 108, p. 131801 (2012).

8. J.K. Ahn et al. (RENO Collab.), Phys.Rev.Lett. 108, p. 191802 (2012).

9. F. P. An et al. (Daya Bay Collab.), Nucl. Instrum. Meth. A811, 133 (2016).

10. F. P. An et al. (Daya Bay Collab.), Phys. Rev. D95, p. 072006 (2017).

11. K. Abe et al. (T2K Collab.), Phys.Rev.Lett. 107, p. 041801 (2011).

12. P. Adamson et al.. (MINOS Collab.), Phys.Rev.Lett. 107, p. 181802 (2011).

13. F.P. An et al. (Daya Bay Collab.), Phys.Rev.Lett. 108, p. 171803 (2012).

14. S. H. Seo et al. (RENO Collab.), arXiv:1610.04326 (2016).

15. P. Adamson et al. (Daya Bay and MINOS Collabs.), Phys. Rev. Lett. 117, p. 151801 (2016), [Addendum: Phys. Rev. Lett.117,no.20,209901(2016)].

16. I. S. Yeo, PoS ICHEP2016, p. 1034 (2017).

17. F. P. An et al. (Daya Bay Collab.), Chin. Phys. C41, p. 013002 (2017).

18. P. Huber, Phys. Rev. C 84, p. 024617 (2011).

19. T. M. et al., Phys. Rev. C 83, p. 054615 (2011).

20. G. Mention et al., Phys.Rev. D83, p. 073006 (2011).

21. H. Seo, Presentation at Applied Antineutrino Physics $201 \%$.

22. Y. Abe et al. (Double CHOOZ Collab.), Phys.Rev. D86, p. 052008 (2012).

23. Y. J. et al. (NEOS Collab.), Phys. Rev. Lett. 118, p. 121802 (Mar 2017).

24. F.P. An et al. (Daya Bay Collab.), Phys. Rev. Lett. 118, p. 251801 (2017).

25. C. Buck, Sterile Neutrinos: Reactor Experiments, in Proceedings, Prospects in Neutrino Physics (NuPhys2016), 2017.

26. F.P. An et al. (JUNO Collab.), J. Phys. G43, p. 030401 (2016).

27. L. J. W. J. Cao and Y. F. Wang, Ann. Rev. Nucl. Part. Sci. 67, 183 (2017).

${ }^{\mathrm{b}}$ FONDECYT is the "Fondo Nacional de Desarrollo Científico, Tecnológico y de Innovación Tecnológica" (National Fund for the Development of Science, Technology, and Technological Innovation). 